\title{
A pilot study to evaluate the feasibility of individualized yoga for inpatient children receiving intensive chemotherapy
}

\author{
Caroline Diorio ${ }^{1}$, Tal Schechter ${ }^{2}$, Michelle Lee ${ }^{1}$, Cathy O'Sullivan ${ }^{1}$, Tanya Hesser ${ }^{1}$, Deborah Tomlinson?',
} Janine Piscione ${ }^{2}$, Christine Armstrong ${ }^{2}$, George Tomlinson ${ }^{3}$ and Lillian Sung ${ }^{1,2^{*}}$

\begin{abstract}
Background: Fatigue is an important problem in paediatric cancer patients and yoga may be an effective intervention. The primary objective was to determine the feasibility of individualized yoga for hospitalized children receiving intensive chemotherapy.

Methods: We included English-speaking children and adolescents aged 7-18 years receiving intensive chemotherapy or haematopoietic stem cell transplantation (HSCT). Yoga was conducted three times weekly for three weeks. The primary outcome was feasibility, defined as ability to deliver at least $60 \%$ of planned sessions. Secondary outcomes were parent-reported Pediatric Quality of Life Inventory (PedsQL) Multidimensional Fatigue Scale, Fatigue Scale-Parent, PedsQL Generic Core Scales and PedsQL Acute Cancer Module.
\end{abstract}

Results: Between January and October 2013, 11 patients were enrolled. Median age was 14.0 (range 7.7-16.4) years and 6 (55\%) were boys. Yoga was feasible with 10/11 participants meeting the threshold for feasibility. The median number of yoga sessions was 9 (range 3-13). No adverse events were attributed to yoga. Mean \pm standard deviation for the day 21 proxy-reported PedsQL general fatigue scores was 55.6 \pm 15.5 . Qualitative comments suggested design changes for future yoga studies.

Conclusions: Individualized yoga is feasible for inpatient children receiving intensive chemotherapy. Future work will include development and conduct of a randomized trial for fatigue amelioration.

Trial registration: ClinicalTrials.gov NCT02105389.

Keywords: Pilot, Yoga, Children, Chemotherapy, Haematopoietic stem cell transplantation, Fatigue, Quality of life

\section{Background}

Cancer-related fatigue can be described as a subjective feeling of physical, emotional, and/or cognitive tiredness [1]. It is thought to be commonly under-recognized, not reported and poorly managed [2]. Further, it is prevalent in children with cancer [3-5]. Three cross-sectional studies conducted in children 10 to 18 years of age found that lack of energy was the most common symptom, occurring in $50 \%$ to $76 \%$ of participants [4,6,7]; fatigue

\footnotetext{
* Correspondence: lillian.sung@sickkids.ca

'Child Health Evaluative Sciences, The Hospital for Sick Children, Peter Gilgan Centre for Research and Learning, 686 Bay St, Toronto, ON M5G 0A4, Canada ${ }^{2}$ Division of Haematology/Oncology, The Hospital for Sick Children, 555 University Ave, Toronto, ON M5G 1X8, Canada

Full list of author information is available at the end of the article
}

symptoms were moderate to very severe in intensity. Fatigue is frequently identified as the most troublesome symptom in paediatric cancer patients with an important impact on quality of life (QoL) [8]. Inpatient status and recent chemotherapy are significant predictors of worse fatigue in children $[7,9]$. Based on severity of illness, anaemia, concurrent medications, inactivity and prolonged inpatient status, children with cancer receiving intensive chemotherapies are expected to be at high risk for severe fatigue.

Guidelines suggest that all cancer patients, including children as young as 5 years of age, should be routinely screened for fatigue [10]. These guidelines also suggest fatigue should be managed according to clinical practice standards. However, evidence demonstrating effective 
interventions for fatigue in children with cancer is scarce. In a recent systematic review of 72 randomized controlled trials (RCTs) which included participants of all ages, exercise reduced fatigue by a moderate amount with a standardized mean difference of -0.45 , 95\% confidence interval $(\mathrm{CI})-0.57$ to $-0.32(\mathrm{P}<0.001)$. Type of exercise (aerobic, walking, resistance, yoga or mixed) did not impact on exercise efficacy (P for interaction $=0.35)$ [11]. Unfortunately, only one study was paediatric-specific.

We hypothesized that yoga would be an ideal intervention for children receiving the most intensive chemotherapy as these children may be too ill to participate in other types of exercise programs. Yoga is a unique intervention that combines exercise and mindfulness. Intensity of the yoga practice can be titrated to a patient's current status and yoga can be delivered in any location without the need for specialized equipment.

In order to evaluate the efficacy of individualized yoga, a RCT is required. However, prior to proceeding to a definitive randomized trial, conduct of a pilot study is important to ensure that a RCT is feasible, to facilitate design of the RCT and to estimate variability of the outcome measure [12]. The primary objective was to determine the feasibility of individualized yoga for hospitalized children receiving intensive chemotherapy. Secondary objectives were to describe: (1) Parent/guardian proxy-report child fatigue scores; (2) Proxy-report child QoL; (3) Self-report parent QoL; (4) Number of children willing to self-report symptoms; and (5) Qualitative suggestions to improve trial conduct.

\section{Methods}

This was a pilot trial of individualized yoga for fatigue in children 7 to 18 years of age receiving intensive chemotherapy at The Hospital for Sick Children (SickKids), Toronto. This study received Research Ethics Board approval from SickKids and all participants/guardians provided informed consent and assent as appropriate.

\section{Subjects}

We included children and adolescents who were: (1) Diagnosed with any acute myeloid leukemia (AML), relapsed acute lymphoblastic leukemia, stage 3 or 4 Burkitt's lymphoma/leukemia or about to receive autologous or allogeneic haematopoietic stem cell transplantation (HSCT); (2) Expected to be an inpatient for at least three weeks after initiation of chemotherapy or conditioning; and (3) Ages 7 to 18 years at enrollment. Exclusion criteria were as follows: (1) Features present to an extent that would preclude compliance with yoga (as assessed by the attending physician): a) motor disability, b) cognitive disability, c) cardiopulmonary symptoms, or d) known compression fracture resulting in disability; and (2) Parent or patient cannot understand English.

\section{Site}

All subjects were recruited from a single site, SickKids in Toronto, Canada. SickKids is the tertiary care referral center for the Greater Toronto area and is the only center that performs paediatric HSCT in the province of Ontario.

\section{Study procedures}

All participants received yoga; yoga began between day -1 to day +4 after starting any course of chemotherapy or conditioning regimens for HSCT. Treatment was administered by one of three trained children's yoga instructors. All yoga instructors were certified teachers in children's yoga and also received study-specific yoga training. It was intended for yoga to occur three times weekly for 3 weeks. However, mid-way through the pilot study, we appreciated that it was difficult to identify 3 appropriate days for yoga in advance because of acuity of illness, scheduling of tests such as computerized tomography scans and other radiological evaluations, and procedures. Consequently, we began to offer yoga daily excluding weekends and holidays (in other words, 4 to 5 times weekly) with the goal of achieving sessions three times weekly.

There was a common structure for all sessions that started with relaxation and breathing followed by a series of poses focused on strengthening, flexibility, and balance. Each session terminated with a period of relaxation (savasana). Yoga instructors were asked to select appropriate poses from a pre-determined list of poses for each session. There were low, moderate and high intensity regimens available depending on the wishes and abilities of the child and parent and the judgment of the yoga instructor. The target intensity was documented and could change with each yoga session. Children were encouraged to choose a focus for each session such as working on a new pose they learned in a previous session, practicing balancing, focusing on deep breathing or focusing on clearing their mind. For each session, the parent or another family member was offered the opportunity to perform yoga along with the child and instructor.

\section{Outcomes}

The primary outcome was feasibility, defined as the ability to deliver at least $60 \%$ of the planned three times weekly yoga sessions in at least $70 \%$ of participants. The time frame evaluated began at the first session and extended until day 21, discharge date or date off study, whichever occurred first. For secondary outcomes, parent proxy-reported child fatigue and QoL were of main interest. We also included child self-reported fatigue and QoL as well as parent self-reported QoL to identify the feasibility and utility of these measures. Parents were the primary respondents while children were encouraged to self-report if they wished. All instruments used a 7 day 
recall period and were administered at baseline and on days 7, 14 and 21 except the Short Form-36 (SF-36) which was administered at baseline and on day 21.

In order to measure fatigue, we included the two instruments with sufficient psychometric evaluation to permit inclusion in trials [13], namely the Pediatric Quality of Life Inventory (PedsQL) Multidimensional Fatigue Scale (MFS) [14] and the Fatigue Scale-Child, Adolescent and Parent (FS-C, FS-A and FS-P) [15]. The PedsQL MFS is an 18-item instrument that assesses general fatigue, sleep/ rest fatigue and cognitive fatigue; it is reliable and valid in children with cancer [14]. We were most interested in general and sleep/rest fatigue. The FS-P is proxy-report and the FS-C (10-items) and FS-A (13-items) are child and adolescent self-report. All scales are reliable, valid and sensitive to change when used longitudinally $[16,17]$.

To measure child QoL, we used the PedsQL 4.0 Generic Core Scale and PedsQL 3.0 Acute Cancer Module. The PedsQL is a multidimensional instrument that is reliable and valid in healthy populations and in children with cancer $[14,18-21]$. From the generic scale, we were most interested in the physical and psychosocial summary scores. From the cancer module, we were most interested in pain and hurt, nausea, anxiety and worry.

In order to measure parent QoL, the Short Form (SF)36 was used. The SF-36 is composed of 36 items that measure physical function, role physical function, bodily pain, general health perception, mental health, role emotional function, energy, and social function [22,23]. Scores for each domain range from 0 to 100, with higher scores indicating better QoL. It results in a physical and a mental health component summary score which have a mean of 50 and standard deviation of 10 , representing the mean and standard deviation of the general United States population. The SF-36 is valid and internally consistent [22,23].

In addition to these quantitative measures, qualitative comments were collected at each assessment time point throughout the study. Participants were asked what they liked and disliked about the yoga program and whether or not they felt the yoga program was of benefit.

\section{Statistical analysis}

Feasibility was defined as the ability to deliver at least $60 \%$ of the planned three times weekly yoga sessions over the evaluation time frame in at least $70 \%$ of participants. We had planned to enroll a minimum of 10 and a maximum of 20 patients to allow modifying the program in the event significant barriers were identified.

\section{Results and discussion}

Between January and October 2013, we assessed 22 potential patients. Of those screened, 5 refused, 6 were missed (not identified during the period of eligibility) and 11 consented to participate. Demographic characteristics are illustrated in
Table 1. Median age was 14.0 (range 7.7-16.4) years and 6 (55\%) were boys. Six children had AML (3 with acute promyelocytic leukemia) and 6 (55\%) were undergoing HSCT. Two children who participated had co-morbidities. One child had ataxia and a compression fracture (not resulting in disability) and one child had cellulitis.

We found that yoga was feasible with $10 / 11$ participants meeting the a priori defined threshold. The median number of yoga sessions was 9 (range 3 to 13). Only one 7 year old child stopped yoga after three sessions because it was not "fast paced" enough for her. No adverse events were experienced, and specifically no musculoskeletal, bleeding or central line issues were attributed to yoga. When the total number of sessions was considered, $26 \%$ were associated with familymember participation. Of the 11 participants, 8 had a family member participate in at least one session. The median age of children who had at least one session with a family member co-participating was 13.5 (range 7 to 16) years. The ages of the child participants without any family member participation were 13, 14 and 16 years.

The proxy-report fatigue scores according to the PedsQL MFS and FS-P are illustrated in Table 2. Proxy-report generic and cancer-specific QoL are also illustrated in Table 2. Only 3 children self-reported fatigue and QoL scores and thus, these scores are not presented. Parent/guardian self-reported QoL according to the SF-36 is illustrated in Table 3.

Qualitative feedback from both children and parents indicated physical and psychological benefits of yoga. Both children and parents noted physical impacts such as increased energy levels, decreased nausea and a

Table 1 Demographics of the study cohort

\begin{tabular}{ll}
\hline Characteristic & Value $\mathbf{N}=\mathbf{1 1}$ \\
\hline Male (\%) & $6(54.6)$ \\
Median age in years (range) & $14.0(7.7-16.4)$ \\
Diagnosis (\%) & \\
$\quad$ Leukemia/lymphoma & $8(72.7)$ \\
Solid tumor & $1(9.1)$ \\
Brain tumor & $1(9.1)$ \\
Aplastic anemia & $1(9.1)$ \\
Median months since diagnosis (IQR) & $2.2(0.2,8.6)$ \\
Relapse status (\%) & $1(9.1)$ \\
Current treatment & \\
Chemotherapy (\%) & $5(45.4)$ \\
Stem cell transplantation (\%) & $6(54.6)$ \\
Parent/other participated in at least one session (\%) & $9(81.8)$ \\
Child previous experience with yoga & $3(27.3)$ \\
\hline
\end{tabular}


Table 2 Proxy report fatigue and quality of life scores*

\begin{tabular}{|c|c|c|c|c|c|c|c|c|}
\hline & \multirow[b]{2}{*}{$\mathbf{N}$} & \multicolumn{2}{|l|}{ Day 0} & \multicolumn{2}{|l|}{ Day 7} & \multicolumn{2}{|l|}{ Day 14} & Day 21 \\
\hline & & Median (IQR) & $\mathbf{N}$ & Median (IQR) & $\mathbf{N}$ & Median (IQR) & $\mathbf{N}$ & Median (IQR) \\
\hline \multicolumn{9}{|l|}{ Fatigue } \\
\hline \multicolumn{9}{|c|}{ PedsQL Multi-dimensional Fatigue Scale } \\
\hline General fatigue & 11 & $50.0(20.8,70.8)$ & 10 & $56.3(35,62.5)$ & 9 & $50.0(33.3,70.8)$ & 9 & $50.0(45.8,70.8)$ \\
\hline Sleep/Rest Fatigue & 11 & $62.5(20.8,66.7)$ & 10 & $41.7(29.2,54.2)$ & 9 & $33.3(29.2,41.7)$ & 9 & $54.2(37.5,66.7)$ \\
\hline Fatigue Scale - Parent & 10 & $40.0(34.0,44.0)$ & 10 & $46.5(44.0,52.0)$ & 10 & $45.5(39.0,49.0)$ & 9 & $44.0(43.0,48.0)$ \\
\hline \multicolumn{9}{|l|}{ Quality of Life } \\
\hline \multicolumn{9}{|l|}{ PedsQL Cancer Module } \\
\hline Pain and hurt & 11 & $50.0(37.5,75.0)$ & 10 & $62.5(25.0,87.5)$ & 7 & $50.0(25.0,75.0)$ & 9 & $75.0(37.5,75.0)$ \\
\hline Nausea & 11 & $50.0(35.0,75.0)$ & 9 & $50.0(35.0,55.0)$ & 7 & $60.0(35.0,80.0)$ & 9 & $50.0(40.0,75.0)$ \\
\hline Procedural Anxiety & 11 & $50.0(25.0,91.7)$ & 9 & $58.3(25.0,100)$ & 6 & $95.8(50.0,100)$ & 9 & $66.7(41.7,100)$ \\
\hline Treatment Anxiety & 11 & $75.0(50.0,100)$ & 9 & $66.7(50.0,100)$ & 6 & $87.5(50.0,100)$ & 9 & $75.0(75.0,100)$ \\
\hline Worry & 11 & $58.3(41.7,83.3)$ & 10 & $62.5(50.0,83.3)$ & 7 & $75.0(50.0,83.3)$ & 9 & $75.0(50.0,83.3)$ \\
\hline \multicolumn{9}{|l|}{ PedsQL Generic Core } \\
\hline Physical health & 11 & $64.3(40.6,71.9)$ & 10 & $48.4(12.5,68.8)$ & 8 & $59.1(34.4,73.4)$ & 6 & $59.9(25.0,75.0)$ \\
\hline Psychosocial health & 11 & $68.8(60.0,81.7)$ & 10 & $62.8(56.7,70.5)$ & 8 & $73.8(65.3,79.6)$ & 9 & $70.0(65.0,80.0)$ \\
\hline
\end{tabular}

Abbreviation: $I Q R=$ interquartile range *Where $\mathrm{N}<11$, evaluations were missing.

reduced need for pain medication. Psychological benefits included reduced anxiety and agitation, better sleep and improved mood. Many children felt yoga had given them the opportunity to relax and 'escape' from the hectic hospital environment.

Several comments will lead to design changes as follows. First, parents felt that four assessments were overly burdensome and that there were too many questionnaires with each assessment. Second, parents noted the social and school functioning questions of the PedsQL Generic Core Scales were not relevant given the inpatient

Table 3 Parent quality of life scores*

\begin{tabular}{lllll}
\hline & & $\begin{array}{l}\text { Day 0 } \\
\text { Nedian (IQR) }\end{array}$ & N & $\begin{array}{l}\text { May 21 } \\
\text { Median (IQR) }\end{array}$ \\
\hline Physical Health Summary & 9 & $47.7(45.0,62.6)$ & 7 & $52.2(41.5,61.0)$ \\
Physical functioning & 11 & $46.5(40.2,57.0)$ & 8 & $52.8(42.3,57.0)$ \\
Role-physical & 10 & $47.1(37.3,56.9)$ & 7 & $54.4(32.4,56.9)$ \\
Bodily pain & 10 & $53.3(41.4,62.1)$ & 8 & $58.8(41.4,62.1)$ \\
General health & 10 & $45.8(38.6,57.7)$ & 8 & $56.5(45.8,62.0)$ \\
Mental Health Summary & 9 & $38.4(33.8,45.9)$ & 7 & $48.1(29.5,56.7)$ \\
Vitality & 11 & $49.0(33.4,58.3)$ & 8 & $44.3(38.1,59.9)$ \\
Social functioning & 11 & $45.9(24.1,51.4)$ & 8 & $48.7(35.0,56.8)$ \\
Role-emotional & 10 & $42.3(36.4,55.9)$ & 8 & $44.3(26.8,55.9)$ \\
Mental health & 10 & $44.4(33.1,50.0)$ & 8 & $50.0(38.7,54.2)$ \\
\hline Abbreviation:
\end{tabular}

Abbreviation: $I Q R=$ interquartile range ${ }^{*}$ Where $\mathrm{N}<11$, evaluations were missing. status of their children. Finally, we found that three times weekly yoga was not an optimal schedule since children may not be available for yoga on a given day related to illness or medical procedures/diagnostic tests. We asked the last 3 children enrolled whether they would participate in a future RCT of yoga if given the opportunity; all 3 said they would participate.

We found that individualized yoga in children receiving intensive chemotherapy is feasible. Our patient population primarily consisted of children with AML and those undergoing HSCT. These children are expected to become profoundly neutropaenic with multiple toxicities of therapy including fever, invasive infection and mucositis and a relatively high treatment-related mortality rate. Consequently, the ability to conduct yoga in this intensively treated and often acutely ill population is very encouraging. We also found that it is feasible to measure fatigue and QoL in these children provided parents are the primary respondents.

In addition, our study provided insight into ways to improve the design of a future RCT. Because of the burdensome nature of outcome assessments, we will reduce the number of assessments to three: baseline, day 10 and day 21. We plan to eliminate the PedsQL Generic Core Scales because of lack of relevance and the SF-36 since caregiver health is not of primary interest, in order to decrease the burden of questionnaire administration. Finally, to improve the ability to deliver yoga, we will change the intervention schedule to daily excluding weekends. Our data also support proxy-response as the 
primary outcome since very few children were willing to self-report symptoms.

Our results are concordant with three other yoga feasibility studies. First, Thygeson and colleagues [24] explored the feasibility of a single yoga session for children 7 to 18 years hospitalized with cancer or other blood disorders. A yoga class was held in the inpatient unit playroom and administered by a researcher who was also a registered yoga teacher. Eleven children participated; the session was feasible and received positive feedback. A second feasibility study of 5 weekly yoga sessions was conducted in 6 children with a variety of cancer types [25]. In this study, a low-impact program was developed by the authors that incorporated elements such as stretching, strengthening and relaxation. The yoga sessions were one hour long, conducted in a group setting, were uniquely adapted to individual patients and were facilitated by physical and occupational therapists, assistants and rehabilitation aides [25]. One child with AML (5 years of age) and two children post HSCT (12 and 19 years of age) were included. The intervention was feasible; children found that they looked forward to participating in the yoga sessions and that the sessions always made them feel better afterwards [25]. A third study demonstrated the feasibility of a 12 week community based program for out-patient children with any type of cancer. Sessions were conducted twice weekly and the authors reported preliminary evidence of program benefits on QoL [26]. Our study is unique, however, as it applies individualized yoga up to 5 times weekly among an intensively treated cancer and HSCT inpatient paediatric population and evaluated the feasibility of measuring specified fatigue and QoL outcomes.

Because of the single armed nature of this pilot study, it is not possible to estimate the effect of yoga on fatigue and QoL outcomes and thus, the definitive RCT will be important to clarify the utility of yoga in this setting. The ability to translate the intervention to a multicenter trial will rely upon careful description of the yoga intervention and training. In our feasibility study, we developed and followed a detailed yoga program and plan to disseminate this information in a subsequent publication. For the future RCT, we plan to provide in-person training to site yoga instructors and to monitor $20 \%$ of yoga sessions remotely to ensure fidelity to the program and safety of the intervention. The future RCT will need to consider the minimally clinically important difference in the fatigue outcome in order to appropriately power the study.

The strengths of this study are the $a$ priori designated definition of feasibility and identification of several key ways to improve the design of the future RCT. However, there are limitations of our study. The most important limitation is that we did not perform randomization within this pilot study and thus, we did not demonstrate the feasibility of enrolling children to a randomized trial. However, all 3 participants who were asked, stated that they would participate in a future RCT of yoga if invited, which provides some reassurance. Second, we performed this pilot study at a single site to ensure we had maximum control of the sessions and outcome ascertainment while we were still in the development phase of the yoga program. A third limitation was the defined threshold for feasibility, namely completion of at least $60 \%$ of the planned three times weekly sessions. This threshold was chosen based on balancing what was likely to be achievable in such an intensively treated population at risk for life-threatening complications and the number of sessions expected to be required to achieve benefit. However, we recognize that this threshold is lower than standards in the exercise field. Another limitation is that we did not define other elements of feasibility, such as duration of sessions, intensity of sessions and completion of required poses. Further research should focus on such elements.

\section{Conclusions}

In summary, individualized yoga is feasible for children with cancer receiving intensive chemotherapy and undergoing HSCT. Future work will proceed toward development and conduct of a RCT of individualized yoga to reduce fatigue in children with cancer and HSCT. Identification of an effective intervention is an important step toward reducing fatigue and improving QoL in this patient population.

\section{Abbreviations}

AML: Acute myeloid leukemia; CRF: Cancer-related fatigue; FS-A: Fatigue scale-adolescent; FS-C: Fatigue scale-child; FS-P: Fatigue scale-parent; HSCT: Haematopoietic stem cell transplantation; MFS: Multidimensional Fatigue Scale; PedsQL: Pediatric Quality of Life Inventory; QoL: Quality of Life; RCT: Randomized Controlled Trial.

\section{Competing interests}

The authors declare that they have no competing interests.

\section{Authors' contributions}

$C D$, TS and LS contributed to the conception of the manuscript. CD, TS, JP, GT and LS contributed to the design of the study. CD, ML, CO, TH, DT, JP and LS were involved in data collection. CD developed the yoga program and $\mathrm{ML}, \mathrm{CO}$ and $\mathrm{TH}$ conducted yoga sessions. CD and LS contributed to data analysis and interpretation of data. CD, TS and LS drafted the manuscript. All authors have contributed to revising the manuscript and have approved the final version of the manuscript.

\section{Acknowledgements}

There is no grant funding for this study.

\section{Author details}

${ }^{1}$ Child Health Evaluative Sciences, The Hospital for Sick Children, Peter Gilgan Centre for Research and Learning, 686 Bay St, Toronto, ON M5G 0A4, Canada. ${ }^{2}$ Division of Haematology/Oncology, The Hospital for Sick Children, 555 University Ave, Toronto, ON M5G 1X8, Canada. ${ }^{3}$ Toronto General Hospital, 200 Elizabeth Street, Toronto, ON M5G 2C4, Canada. 
Received: 5 February 2014 Accepted: 16 January 2015

Published online: 24 January 2015

\section{References}

1. Mortimer JE, Barsevick AM, Bennett CL, Berger AM, Cleeland C, Devader SR, et al. Studying Cancer-Related Fatigue: Report of the NCCN Scientific Research Committee. J Natl Compr Canc Netw. 2010;8(12):1331-9.

2. Mitchell SA. Cancer-related fatigue: state of the science. PM R. 2010;2 (5):364-83.

3. Erickson JM, Beck SL, Christian B, Dudley WN, Hollen PJ, Albritton K, et al. Patterns of fatigue in adolescents receiving chemotherapy. Oncol Nurs Forum. 2010;37(4):444-55.

4. Baggott C, Dodd M, Kennedy C, Marina N, Matthay KK, Cooper BA, et al. Changes in children's reports of symptom occurrence and severity during a course of myelosuppressive chemotherapy. J Pediatr Oncol Nurs. 2010;27 (6):307-15.

5. Dupuis LL, Milne-Wren C, Cassidy M, Barrera M, Portwine C, Johnston DL, et al. Symptom assessment in children receiving cancer therapy: the parents' perspective. Support Care Cancer. 2010;18(3):281-99.

6. Walker AJ, Gedaly-Duff V, Miaskowski C, Nail L. Differences in symptom occurrence, frequency, intensity, and distress in adolescents prior to and one week after the administration of chemotherapy. J Pediatr Oncol Nurs. 2010;27(5):259-65

7. Collins JJ, Byrnes ME, Dunkel IJ, Lapin J, Nadel T, Thaler HT, et al. The measurement of symptoms in children with cancer. J Pain Symptom Manage. 2000;19(5):363-77.

8. Gibson F, Garnett M, Richardson A, Edwards J, Sepion B. Heavy to carry: a survey of parents' and healthcare professionals' perceptions of cancer-related fatigue in children and young people. Cancer Nurs. 2005;28(1):27-35.

9. Collins JJ, Devine TD, Dick GS, Johnson EA, Kilham HA, Pinkerton CR, et al. The measurement of symptoms in young children with cancer: the validation of the Memorial Symptom Assessment Scale in children aged 7-12. J Pain Symptom Manage. 2002;23(1):10-6.

10. Berger AM, Mooney K, Alvarez-Perez A, Breitbart WS, Carpenter KM, Cella D, et al. National Comprehensive Cancer Network Clinical Practice Guidelines in Oncology: Cancer-Related Fatigue (Version 1.2015). Retrieved from http://www. nccn.org/professionals/physician_gls/f_quidelines.asp\#fatigue.

11. Tomlinson D, Diorio C, Beyene J, Sung L: The effect of exercise on cancer-related fatigue. A meta-analysis. Am J Phys Med Rehabil. in press.

12. Thabane L, Ma J, Chu R, Cheng J, Ismaila A, Rios LP, et al. A tutorial on pilot studies: the what, why and how. BMC Med Res Methodol. 2010;10:1.

13. Tomlinson D, Hinds PS, Ethier MC, Ness KK, Zupanec S, Sung L. Psychometric Properties of Instruments Used to Measure Fatigue in Children and Adolescents With Cancer: A Systematic Review. J Pain Symptom Manage. 2013;45(1):83-91.

14. Varni JW, Burwinkle TM, Katz ER, Meeske K, Dickinson P. The PedsQL in pediatric cancer: reliability and validity of the Pediatric Quality of Life Inventory Generic Core Scales, Multidimensional Fatigue Scale, and Cancer Module. Cancer. 2002;94(7):2090-106.

15. Hinds PS, Yang J, Gattuso JS, Hockenberry M, Jones H, Zupanec S, et al. Psychometric and clinical assessment of the 10-item reduced version of the Fatigue Scale-Child instrument. J Pain Symptom Manage. 2010;39(3):572-8.

16. Mandrell BN, Yang J, Hooke MC, Wang C, Gattuso JS, Hockenberry M, et al. Psychometric and clinical assessment of the 13-item reduced version of the fatigue scale-adolescent instrument. J Pediatr Oncol Nurs. 2011;28(5):287-94.

17. Hinds PS, Hockenberry M, Tong X, Rai SN, Gattuso JS, McCarthy K, et al. Validity and reliability of a new instrument to measure cancer-related fatigue in adolescents. J Pain Symptom Manage. 2007;34(6):607-18.

18. Varni JW, Katz ER, Seid M, Quiggins DJ, Friedman-Bender A. The pediatric cancer quality of life inventory-32 (PCQL-32): I. Reliability and validity. Cancer. 1998:82(6):1184-96.

19. Varni JW, Katz ER, Seid M, Quiggins DJ, Friedman-Bender A, Castro CM. The Pediatric Cancer Quality of Life Inventory (PCQL). I. Instrument development, descriptive statistics, and cross-informant variance. J Behav Med. 1998;21 (2):179-204.

20. Varni JW, Rode CA, Seid M, Katz ER, Friedman-Bender A, Quiggins DJ. The Pediatric Cancer Quality of Life Inventory-32 (PCQL-32). II. Feasibility and range of measurement. J Behav Med. 1999;22(4):397-406.

21. Varni JW, Seid M, Kurtin PS. PedsQL 4.0: reliability and validity of the Pediatric Quality of Life Inventory version 4.0 generic core scales in healthy and patient populations. Med Care. 2001;39(8):800-12.
22. Brazier JE, Harper R, Jones NM, O'Cathain A, Thomas KJ, Usherwood T, et al. Validating the SF-36 health survey questionnaire: new outcome measure for primary care. BMJ. 1992;305(6846):160-4.

23. Jenkinson C, Wright L, Coulter A. Criterion validity and reliability of the SF-36 in a population sample. Qual Life Res. 1994;3(1):7-12.

24. Thygeson MV, Hooke MC, Clapsaddle J, Robbins A, Moquist K. Peaceful play yoga: serenity and balance for children with cancer and their parents. J Pediatr Oncol Nurs. 2010;27(5):276-84.

25. Geyer R, Lyons A, Amazeen L, Alishio L, Cooks L. Feasibility study: the effect of therapeutic yoga on quality of life in children hospitalized with cancer. Pediatr Phys Ther. 2011;23(4):375-9.

26. Wurz A, Chamorro-Vina C, Guilcher GM, Schulte F, Culos-Reed SN. The feasibility and benefits of a 12-week yoga intervention for pediatric cacner out-patients. Pediatr Blood Cancer. 2014;61(10):1828-34.

\section{Submit your next manuscript to BioMed Central and take full advantage of:}

- Convenient online submission

- Thorough peer review

- No space constraints or color figure charges

- Immediate publication on acceptance

- Inclusion in PubMed, CAS, Scopus and Google Scholar

- Research which is freely available for redistribution 